\title{
Antiviral effect of 1-calcium phosphonate uracil against porcine reproductive and respiratory syndrome virus in vitro
}

Lei Xu (D601473235@qq.com)

Sichuan agricultural University https://orcid.org/0000-0001-9554-6927

Zhi wen Xu

Sichuan Agricultural University

Zhao Jun

SiChuan agricultural university

Xiao yu Yang

Sichuan agriculture University

Ruo bo Zhang

Sichuan agriculture University

Chao yuan Jiang

Sichuan agriculture University

Feng Yu

Sichuan agriculture University

Xin huan Yin

Sichuan agriculture University

Fei Li

Sichuan agriculture University

Jianbo Huang

Sichuan agriculture University

Xiangang Sun

Sichuan agriculture University

Zhu Ling

Sichuan agriculture University

Research article

Keywords: PRRSV, uracil, antiviral

Posted Date: December 13th, 2019

DOI: https://doi.org/10.21203/rs.2.17719/v2 
License: (c) (i) This work is licensed under a Creative Commons Attribution 4.0 International License. Read Full License 


\section{Abstract}

Background: Porcine reproductive and respiratory syndrome virus (PRRSV) is an RNA virus. Currently, it is treated in various ways, including vaccination with inactivated vaccine, live attenuated vaccine, or subunit vaccine, but the control of PRRSV infection is still inadequate. Therefore, it is necessary to study new drugs against PRRSV infection. In this study, the antiviral effect of 1-calcium phosphonate uracil on PRRSV in vitro was studied. Results: PRRSV was significantly inhibited in SR pretreated cells. In addition, the results showed that SR inhibited the adhesion, entry and genome synthesis of PRRSV, but did not affect the release of PRRSV. Conclusion: Our results suggest that SR may play an antiviral role in PRRSV infection in vitro, which suggests that SR may be a potential new drug for inhibiting PRRSV infection

\section{Background}

Porcine reproductive and respiratory syndrome (PRRS) has been one of the major infectious diseases threatening the healthy development of the pig industry. The clinical signs mainly include abortion, premature delivery, stillbirth and mummification in pregnant sows, and piglets and growing pigs present with respiratory symptoms accompanied by interstitial pneumonia. PRRSV is a single-strand positivesense RNA virus belonging to the arteritis virus family, which can be divided into two genotypes: the European LV strain and North American VR2332 strain[1, 2]. PRRSV can induce immunosuppression and cause persistent infection, which makes preventing and controlling the disease difficult[3]. Previous studies have reported that many natural compounds, microRNAs and new vaccinations can control PRRSV infection[4-6]. However, despite the wide use of the current vaccination strategy, the protection against PRRSV infection remains limited. Therefore, it is necessary to develop a safe and effective antiviral strategy. 1-Calcium phosphate-uracil (SR) is a derivative of uracil and is a phosphorylated nitrogenous base drug (Fig. 1A). Its molecular formula is $\mathrm{C}_{4} \mathrm{H}_{3} \mathrm{O}_{5} \mathrm{NPCa}$, and its molecular weight is $216.1276 \mathrm{~g} / \mathrm{mol}$. Recent clinical research has demonstrated that the drug has multiple significant effects, including antiviral, antibacterial toxic infection, and tumour inhibition effects.

In this study, we investigated the antiviral effect of SR on PRRSV infection in vitro. We found that PRRSV replication is effectively inhibited by SR. In addition, because the replication of the virus genome in host cells is inhibited, SR has antiviral activity against PRRSV in vitro. In summary, SR has high value for clinical application, because it may be useful in antiviral treatment of PRRSV infection.

\section{Results}

\section{The growth of PRRSV was significantly inhibited in Marc-145 treated with SR}

To analyse whether SR has antiviral activity against PRRSV, the following experiments were carried out. First, the cytotoxicity induced by SR in MARC-145 cells was tested using an MTT cell proliferation and cytotoxicity assay kit. Compared with the impact using the mock treatment, none of the different concentrations of SR had an obvious impact on the viability of MARC-145 cells, as measured by the 
absorbance of cell cultures at $570 \mathrm{~nm}$ (Fig. 1B). Then, we investigated the inhibitory effect of SR on PRRSV by indirect immunofluorescence experiments. The results showed that, compared with the proliferation of PRRSV in the PBS group, the proliferation of PRRSV was inhibited at different concentrations of SR-treatments applied to the Marc-145 cells, indicating that SR significantly inhibited the proliferation of PRRSV (Fig. 1C).

To explore the antiviral function of SR, we infected Marc-145 cells with a PRRSV DJY and SR mixture and cultured them at $37^{\circ} \mathrm{C}$ for two hours. Then, we washed the cells with PBS, removed the non-adsorbed virus particles, added $2 \%$ fresh DMEM containing $100 \mu \mathrm{M}$ SR, and measured the relative expression of ORF7 mRNA with qRT-PCR $48 \mathrm{hpi}$. The results showed that, compared with the PRRSV replication after the mock treatment, SR significantly and continuously inhibited the replication of PRRSV (Fig. 1D). In the presence of different concentrations of SR $(0,10,20,40,60,80$ and $100 \mu \mathrm{M})$, compared with the untreated PRRSV-infected Marc-145 cells, the virus titre was decreased, through dose-dependent inhibition (Fig. 1E).

\section{The proliferation of PRRSV was inhibited in SR-pretreated Marc-145 cells}

Marc-145 cells were pretreated with $100 \mu \mathrm{M}$ SR or PBS for $24 \mathrm{~h}$, and then infected with PRRSV DJY at a dose of $\mathrm{MOI}=1$. The virus titres of the Marc-145 cells pretreated with SR were measured 12, 24, 36 and 48 hpi. Compared with that of the PBS group, the virus titre of Marc-145 cells pretreated with SR was decreased (Fig. 2A). These results showed that PRRSV DJY proliferation in the SR pretreated cells was affected, but in the later stage, the inhibition decreased gradually with prolonged exposure times.

We examined whether SR can inactivate PRRSV infection. PRRSV DJY $(\mathrm{MOI}=0.1)$ was mixed with 100 $\mu \mathrm{M}, 10 \mu \mathrm{M}$, and $1 \mu \mathrm{M} \mathrm{SR}$, and the mixture was incubated at room temperature for $2 \mathrm{~h}$, and then used to infect Marc-145 cells in a 96-well plate. The virus titre was determined after $48 \mathrm{dpi}$. As shown in Figure $2 B$, compared with the effect of the PBS treatment, the SR treatment with concentrations of $100 \mu \mathrm{M}, 10$ $\mu \mathrm{M}$ and $1 \mu \mathrm{M}$ had no significant effect on the PRRSV titre, indicating that SR could not directly inactivate PRRSV infectivity.

\section{PRRSV attachment and entry were significantly inhibited by SR}

In order to further determine the precise mechanism of SR inhibition in the virus life cycle, we first evaluated the effect of SR on PRRSV adsorption. Precooled Marc-145 cells were inoculated with a mixture of PRRSV DJY and SR $(100 \mu \mathrm{M})$ with different $\mathrm{MOI}$ ratios $(\mathrm{MOI}=10,1,0.1$, or 0.01$)$ and then incubated at $4^{\circ} \mathrm{C}$ for another $2 \mathrm{~h}$. The cells were washed with PBS to remove the non-adsorbed virus particles and then incubated at $37^{\circ} \mathrm{C}$, and the virus titre was measured $48 \mathrm{hpi}$. As shown in Figure $3 \mathrm{~A}$, compared with the PRRSV proliferation after PBS treatment, the SR treatment significantly inhibited the proliferation of PRRSV at each infection dose, indicating that SR may inhibit the adsorption of PRRSV at the cell surface, but the effect was not obvious. 
To further explore the effect of SR on PRRSV entry, PRRSV DJY was allowed to adsorb on precooled Marc- 145 cells at $4^{\circ} \mathrm{C}$ at different MOls. SR $(100 \mu \mathrm{M})$ was then added to the virus-adsorbed cells, and the cells were incubated at $37^{\circ} \mathrm{C}$. After incubation for $3 \mathrm{~h}$, the medium containing SR was replaced with fresh medium, and the cells were cultured at $37^{\circ} \mathrm{C}$ for another $48 \mathrm{~h}$ to determine the virus titre. The results showed that the virus titre of the Marc-145 cells treated with SR decreased approximately 5 -fold when infected with virus between 10 and $0.01 \mathrm{MOI}$ compared with titre of the PBS-treated Marc-145 cells (Fig. 3B). In conclusion, these results suggest that SR inhibits PRRSV entry into Marc- 145 cells.

\section{SR had a significant effect on PRRSV genome synthesis}

To determine whether SR inhibits PRRSV genome synthesis, we analysed the effect of SR on PRRSV genome synthesis. Marc- 145 cells were infected with PRRSV DJY $(\mathrm{MOl}=1)$ at $37^{\circ} \mathrm{C}$ for $2 \mathrm{~h}$, and then were treated with SR $(100 \mu \mathrm{M})$ or PBS. Samples were taken at the designated time points $(12 \mathrm{~h}, 24 \mathrm{~h}$, and $36 \mathrm{~h})$ to prepare total RNA and analyse the PRRSV genome by real-time RT-PCR. The results showed that the relative level of GP7 in the Marc-145 cells treated with SR was significantly reduced compared with the level in the PBS at all times, and the effect followed a time gradient. Twelve hours after treatment, the relative level of PRRSV was reduced by 10 -fold (Fig. $3 C$ ), indicating that SR could significantly inhibit the synthesis of the PRRSV genome.

To determine whether SR can inhibit the release of PRRSV, Marc-145 cells were infected with PRRSV DJY (MOI=1). Twenty-four hours post-infection, the inoculum was replaced with fresh medium containing SR. The virus titres in the supernatant were measured 15 minutes, 30 minutes, 45 minutes and 60 minutes after the medium was replaced. As shown in Figure 3D, in the supernatant, the PRRSV titre of the cells treated with SR did not decrease significantly at the different time points, compared with the PRRSV titre in the PBS-treated cells, indicating that SR had no effect on the release of PRRSV virus particles.

\section{Discussion}

Currently, most antiviral drugs on the market are purine derivatives or pyrimidine derivatives, such as acyclovir and valaciclovir, which are DNA polymerase inhibitors. They can inhibit the thymidine kinase and DNA polymerases encoded by the virus; hence, they can significantly inhibit the synthesis of DNA in infected cells without affecting the DNA replication of the non-infected cells[7]. Most uracil analogues affect protein translation by interfering with DNA/RNA synthesis. For example, as an anticancer drug, fluorouracil is used in the treatment of gastrointestinal cancer, breast cancer, rectal cancer, etc., by inhibiting thymine nucleotide synthetase, thereby blocking the conversion of deoxypyrimidine nucleotides into thymine nucleosides, it interferes with DNA synthesis[8, 9]. The results from the latest research indicated that the nucleoside derived rigid amphiphilic fusion inhibitors (Rafis), 5-(perylen-3-ylethynyl) arabino uridine (auy11) and 5- (perylen-3-ylethynyl) uracil-1-ac acid (cm1uy11), are active against African swine fever virus (ASFV). Rafis stabilize the positive curvature of the membrane through its molecular structure, thus inhibiting the fusion of the virus with the cell[10]. In addition, researchers have confirmed that Rafis can significantly inhibit virus adhesion, but duy11, another member of Rafi familys, has no 
effect on virus adhesion, which may be due to different substituents leading to different molecular characteristics, for example, the 1-benzoyl-3-[(4,6-dimethyl-2-yl) methyl] uracil group and the 1-(2,6difluorobenzyl-3-[(2,6-dimethyl-4-yl) methyl-3-[(2,6-dimethyl-4-yl) methyl group, through a pyrenyl moiety, had significantly reduced activity against HCMV[11]. All of these results indicate that most of the antiviral effects caused by the uracil derivatives occur in cells. However, our research results show that SR can also inhibit the absorption and entry of the virus, which may be caused by the absorption of SR by the cells incubated with of SR, such that the virus that enters the cells is inhibited by SR during the process of proliferation. As everyone knows, the pyrimidines needed for RNA transcription are all synthesized from scratch, so uracil derivatives cannot inhibit virus proliferation by participating in the synthesis of virus RNA. However, the results show that the main inhibition period of virus genome synthesis; therefore, the specific antiviral function of SR remains to be further studied.

At present, there is still a view that when the concentration of $\mathrm{Ca}^{2+}$ in cells is too high, the $\mathrm{Ca}^{2+}$-dependent protease CaMMK $\beta$ and its substrate molecules AMPK and CaMKI may be activated, which may lead to the initiation of autophagy[12]. Therefore, we speculate that SR is degraded into intracellular $\mathrm{Ca}^{2+}$, which would lead to an increase in intracellular $\mathrm{Ca}^{2+}$ concentrations and thus induce autophagy in the cells. Of course, this speculation has yet to be confirmed.

\section{Conclusion}

In this study, we found that SR has obvious anti PRRSV effect and that it mainly affects the synthesis of the PRRSV genome, suggesting a new treatment method and a new perspective for the treatment of PRRSV infection.

\section{Methods}

\section{Cells, viruses and reagents}

MARC-145 cells were grown in Dulbecco's modified Eagle medium (DMEM) (Fisher Scientific) supplemented with 10\% FBS (Sangon Biotech, Shanghai, China). The stock of an NADC30-like PRRSV DJY and an HP-PRRSV NJ virus were isolated and stored by our laboratory for use in this study. 1-

Calcium phosphonate uracil was donated by Sichuan Huiling, Xinkang Technology Development Co., Ltd. (Sichuan Chengdu, China).

HRP-conjugated goat anti-rabbit IgG (catalogue no: D110058) was purchased from Sangon Biotech (Shanghai, China). Rabbit monoclonal antibody (mAb) against PRRSV was prepared in our laboratory. A TRIzol reagent kit (catalogue no: 206101) was purchased from Magen (Beijing, China). TB Green Premix Ex Taq II (Tli RNaseH Plus) (catalogue no: RR820Q) and the PrimeScript ${ }^{\text {TM }}$ RT reagent kit (Perfect Real Time) (RR037A) were purchased from TaKaRa (Beijing, China).

\section{Cell viability assay}


The impacts of SR on MARC-145 cell viability were examined using a MTT cell proliferation and cytotoxicity assay kit (MTT) (Sangon Biotech, Shanghai, China).

For the cell cytotoxicity assay, MARC-145 cells were seeded at a density of 5000 cells/well in $100 \mu \mathrm{L}$ of cell culture medium in a 96-well plate. The MARC-145 cells were incubated in these plate wells for $24 \mathrm{~h}$ in a $\mathrm{CO} 2$ incubator before treatment. After treatment with different concentrations of SR for $48 \mathrm{~h}$, we added $10 \mu \mathrm{L}$ of MTT reagent (prepared as described above) at a final concentration of $0.5 \mathrm{mg} / \mathrm{mL}$ to each well. The compound was mixed gently and incubated at $37^{\circ} \mathrm{C}$ for $4 \mathrm{~h}$ in a $\mathrm{CO} 2$ incubator. We aspirated the culture medium from each well carefully to prevent disruption of the cell monolayer. Then, $100 \mu \mathrm{L}$ of formazan solubilization solution was added to each well. The plate was gently mixed on a shaker for 10 minutes to dissolve the formazan crystals. Then, the absorbance was measured at $570 \mathrm{~nm}$ using a microplate reader.

\section{Virus infection and titration}

Marc-145 cells were grown in 96-well culture plates. The virus was diluted on a 10-fold gradient $\left(10^{-1}-10^{-}\right.$ ${ }^{10}$ ) and then inoculated into the cultures in the 96 -well culture plate. After $48 \mathrm{~h}$ of infection, the $\mathrm{TCID}_{50}$ of the PRRSV was calculated by the Reed-Muench method.

\section{Indirect immunofluorescence assay (IFA)}

The MARC-145 cells infected with PRRSV were fixed with $4 \%$ paraformaldehyde for one night at $4^{\circ} \mathrm{C}$, blocked for 60 minutes with $5 \%$ bovine serum albumin (BSA), treated with monoclonal antibodies against the GP4 protein of PRRSV, incubated with FITC-conjugated goat anti-mouse IgG for 60 minutes, and finally observed under a fluorescence microscope (Nikon, Japan).

\section{Real-time RT-PCR}

Total RNA was extracted from the infected MARC-145 cells using TRIzol reagent according to the manufacturer's manual. The isolated RNA was reverse-transcribed using the PrimeScript RT reagent kit as described above. A pair of primers specific for the glycosylated membrane protein 7 (GP7)-coding region of PRRSV was used to examine the PRRSV genomic RNA after real-time PCR with TB Green Premix Ex Taq II. Real-time PCR was performed in a LightCycler® 96 Real-Time PCR system (Roche, Switzerland). The CDNA that was obtained was pre-denatured at $94^{\circ} \mathrm{C}$ for $30 \mathrm{~s}$, followed by 40 cycles of amplification at $95^{\circ} \mathrm{C}$ for $5 \mathrm{~s}, 60^{\circ} \mathrm{C}$ for $30 \mathrm{~s}$ and $72^{\circ} \mathrm{C}$ for $30 \mathrm{~s}$ with final melting at $95^{\circ} \mathrm{C}$ for $5 \mathrm{~s}, 65^{\circ} \mathrm{C}$ for $60 \mathrm{~s}$ and $97^{\circ}$ $\mathrm{C}$ for $1 \mathrm{~s}$. Cellular $\beta$-actin in the MARC-145 cells was quantified as the internal control. The gene-specific primer sequences were as follows: 5'- AGCCAGTCCAGAGGTAAGGG -3' (forward) and 5'- CGC CTT GGT TAA AGG CAG TC -3' (reverse) for the GP7-coding region and 5'- GGG ACC TGA CCG ACT ACC TCA TG -3' (forward) and 5'- GCC ATC TCC TGC TCG AAG TCC AG -3' (reverse) for $\beta$-actin. The $2^{\wedge}-\Delta \triangle C$ t method was used to calculate the relative levels of the viral genomic RNA.

\section{Viral attachment assay}


MARC-145 cells grown on 12-well plates were pre-chilled at $4^{\circ} \mathrm{C}$ for $1 \mathrm{~h}$ and then inoculated with the mixture of PRRSV at the different MOls and SR $(100 \mu \mathrm{M})$ and were incubated at $4^{\circ} \mathrm{C}$ for an additional $2 \mathrm{~h}$. The mixture of the virus and SR was then removed, and the cell monolayers were washed three times with pre-chilled PBS before fresh medium was added. After further incubation at $37^{\circ} \mathrm{C}$ for $48 \mathrm{~h}$, the virus titres were measured as described above.

\section{Viral penetration assay}

Monolayers of the MARC- 145 cells were pre-chilled at $4^{\circ} \mathrm{C}$ for $1 \mathrm{~h}$ and then infected with the virus at different $\mathrm{MOI}$ ratios for $2 \mathrm{~h}$ at $4^{\circ} \mathrm{C}$. The virus-containing medium was removed, and the cells were washed three times with pre-chilled PBS prior to treatment with medium containing SR $(100 \mu \mathrm{M})$, in which they were incubated for $3 \mathrm{~h}$ at $37^{\circ} \mathrm{C}$. The cells were carefully washed with PBS to remove the SR and noninternalized extracellular virus and then fresh medium was added to them. After further incubation at $37^{\circ} \mathrm{C}$ for $48 \mathrm{~h}$, the virus titres were measured as described above.

\section{Viral synthesis assay}

Marc- 145 cells were pre-cooled for $1 \mathrm{~h}$ at $4^{\circ} \mathrm{C}$ and then infected with virus at an MOl=1 and $4^{\circ} \mathrm{C}$ for $2 \mathrm{~h}$. The virus-containing medium was removed. The virus was washed with pre-cooled PBS 3 times, and them the non-internalized extracellular virus was removed. The cells were treated with SR $(100 \mu \mathrm{M})$ medium at $37^{\circ} \mathrm{C}$ for $6 \mathrm{~h}, 12 \mathrm{~h}, 24 \mathrm{~h}, 36 \mathrm{~h}$ or $48 \mathrm{~h}$. Fluorescence quantitative PCR was performed according to the methods described above.

\section{Viral release assay}

MARC-145 cell monolayers were infected with PRRSV (MOI=1). Twenty-four hours post-infection (hpi), the inoculated medium was replaced by medium containing SR $(100 \mu \mathrm{M})$, and then, 15 minutes, 30 minutes, 45 minutes and 60 minutes later, both the supernatant and the infected cells were harvested separately and titrated as described above.

\section{Statistical analysis}

The data were expressed as the means \pm standard deviations (SD). The differences among different groups were analysed for significance using two-way ANOVA in GraphPad Prism software (version 5.0). Differences were considered significant at a $p$ value $<0.05$.

\section{Abbreviations}

PRRS: Porcine reproductive and respiratory syndrome; SR:1-calcium phosphate-uracil; MOI: Multiplicity of infection

\section{Declarations}




\section{Ethics approval and consent to participate}

Not applicable.

\section{Consent for publish}

Not applicable.

\section{Availability of data and materials}

The datasets used and/or analysed during the current study are available from the corresponding author on reasonable request.

\section{Competing interests}

All authors have no competing interests to report.

\section{Funding}

This research was funded by National Key Research Program during the 13th Five-year Plan Period of China: Integrated Prevention and Control Technology for Major Diseases of Commercial Pigs and Integration of Safe Drug Use Technology(2018YFD0501102), Research and Demonstration of Purification Technology of Pseudorabies(2018YFD0500802). The Program Sichuan Veterinary Medicine and Drug Innovation Group of China Agricultural Research System (CARS-SVDIP).

\section{Authors' contributions}

ZL and XLconceived the study. ZL and ZWX designed the experiments. $X L, Z J, X Y Y, R B Z, C Y J, F Y, X H Y$ and LF performed the experiments. $\mathrm{XL}, \mathrm{JBH}$ and $\mathrm{XGS}$ wrote the manuscript. All authors read and approved the final manuscript.

\section{Acknowledgements}

The author would like to thank Dr. Lin for providing SR drugs.

\section{Authors' information (optional)}

${ }^{1}$ College of veterinary medicine, Sichuan Agricultural University, Chengdu, Sichuan, China.

${ }^{2}$ Key Laboratory of Animal Diseases and H $\mu$ Man Health of Sichuan Province, Sichuan Agricultural University, Chengdu, Sichuan, China.

Xu Lei, College of Veterinary Medicine, Sichuan Agricultural University, Huimin Road 211\#, Wenjiang District, Chengdu, 611130, Sichuan Province, China. Email: 601473235@qq.com 


\section{References}

1. Wensvoort G, Terpstra C, Pol JMA, ter Laak EA, Bloemraad M, de Kluyver EP, Kragten C, van Buiten L, den Besten A, Wagenaar F. Mystery swine disease in the Netherlands: The isolation of Lelystad virus. Vet Q, 1991;13(3):121-30.

2. Benfield DA, Nelson E, Collins JE, Harris L, Goyal SM, Robison D, Christianson WT, Morrison RB, Gorcyca D, Chladek D. Characterization of Swine Infertility and Respiratory Syndrome (SIRS) Virus (Isolate ATCC VR-2332). J Vet Diagn Invest. 1992;4(2):127-33.

3. Rowland RR. The stealthy nature of PRRSV infection: The dangers posed by that ever-changing mystery swine disease. Vet J. 2007;174(3):451.

4. Li L, Wei Z, Zhou Y, Gao F, Jiang Y, Yu L, Zheng H, Tong W, Yang S, Zheng H. Host miR-26a suppresses replication of porcine reproductive and respiratory syndrome virus by upregulating type $\mathrm{I}$ interferons. Virus Res. 2015;195:86-94.

5. Hao H-p, Wen L-b, Li J-r, Wang Y, Ni B, Wang R, Wang X, Sun M-X, Fan H-j, Mao X. LiCl inhibits PRRSV infection by enhancing Wnt/ $\beta$-catenin pathway and suppressing inflammatory responses. Antiviral Res. 2015;117:99-109.

6. Wan B, Bao DK, Qiao SL, Xu D, Yang SZ, Wang YB, Zhang GP. Expression of porcine interferon-a and detection of its antiviral activity against PRRSV in PAM. J Henan Agr Sci, 2010;(3):93-9.

7. Wagstaff AJ, Faulds D, Goa KL. Aciclovir. Drugs.1994;47(1):153-205.

8. Shinkai I, Ohta Y. Valacyclovir. Bioorg Med Chem.1996;4(1):1-2.

9. Duschinsky R, Pleven E, Heidelberger C. THE SYNTHESIS OF 5-FLUOROPYRIMIDINES. J. Am. Chem. Soc. 1957;79(16):4559-60.

10. Hakobyan A, Galindo I, Nañez A, Arabyan E, Zakaryan H. Rigid amphipathic fusion inhibitors demonstrate antiviral activity against African swine fever virus. J Gen Virol. 2018;99(1):148-56.

11. T M, Y D, S K, M W, C P, J B, R S, G A, E DC. Antiviral activity of 3-(3,5-dimethylbenzyl)uracil derivatives against HIV-1 and HCMV. Nucleosides Nucleotides Nucleic Acids. 2007;26(10-12):1553-8.

12. Gu Y, Baozhu Q, Yingshan Z, Xiaowu J, Xian Z, Xiaoliang L, Weihuan F. Porcine Circovirus Type 2 Activates CaMMK $\beta$ to Initiate Autophagy in PK-15 Cells by Increasing Cytosolic Calcium. Viruses. 2016;8(5):135-52.

\section{Figures}


C
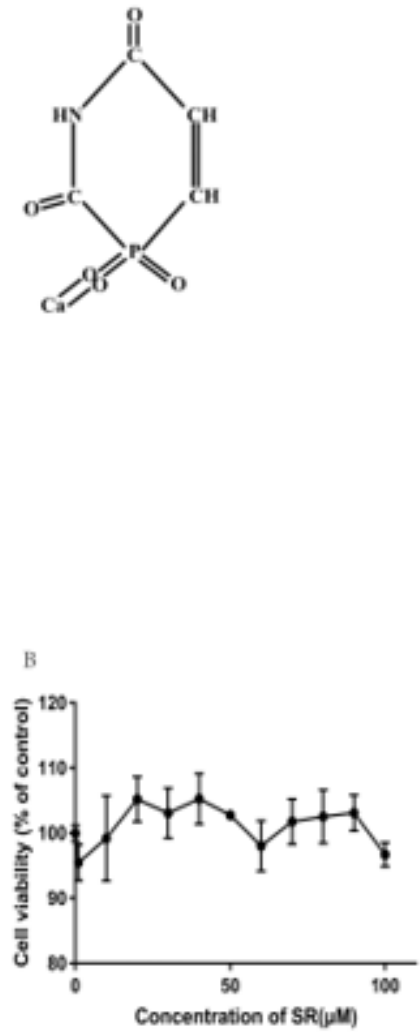
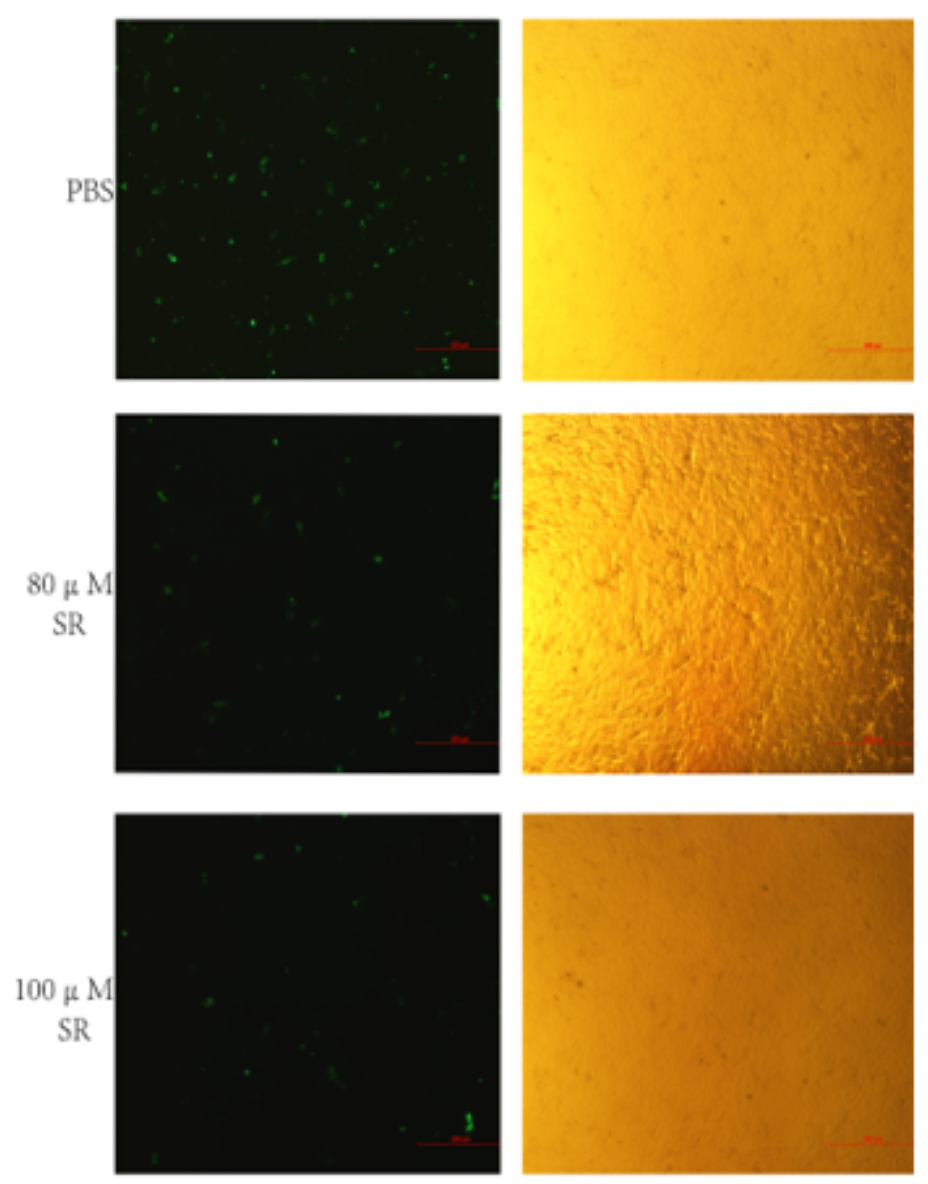
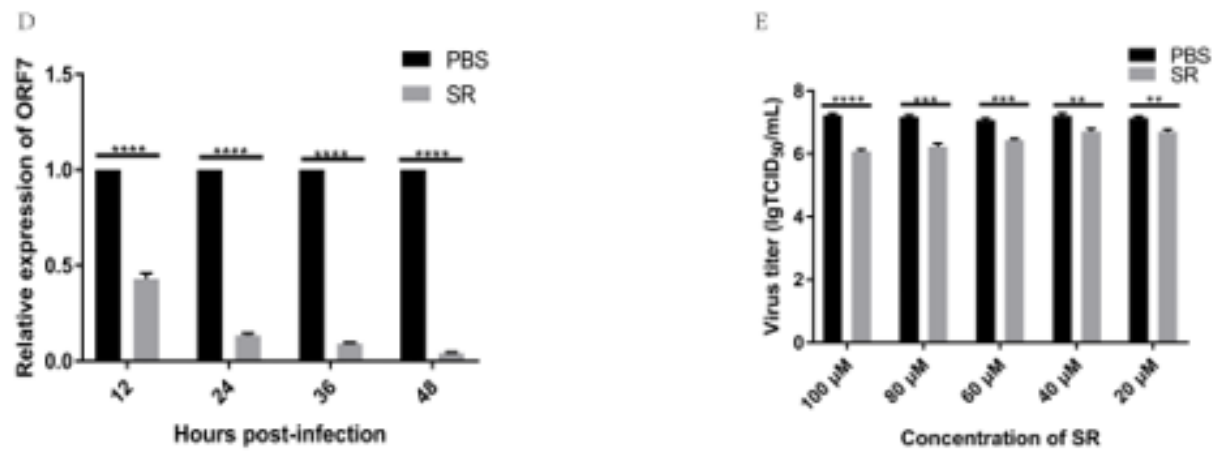

\section{Figure 1}

The growth of PRRSV was inhibited by SR. The structural formula of SR (A). MTT was used to detect the toxicity induced by SR in the Marc-145 cells (B). The inhibitory effect of SR on PRRSV was observed by IFA (C). At different times (12 h, $24 \mathrm{~h}, 36 \mathrm{~h}$, and $48 \mathrm{~h})$ and at different concentrations $(0,10,20,40,60,80$ and $100 \mu \mathrm{M}$ ), the SR inhibited PRRSV (D and E). Data are shown as the means \pm SD of three independent experiments ( ${ }^{*} p<0.05,{ }^{* *} p<0.01,{ }^{* * *} p<0.001$, and ${ }^{* * * *} p<0.001$; ns, not significant). 

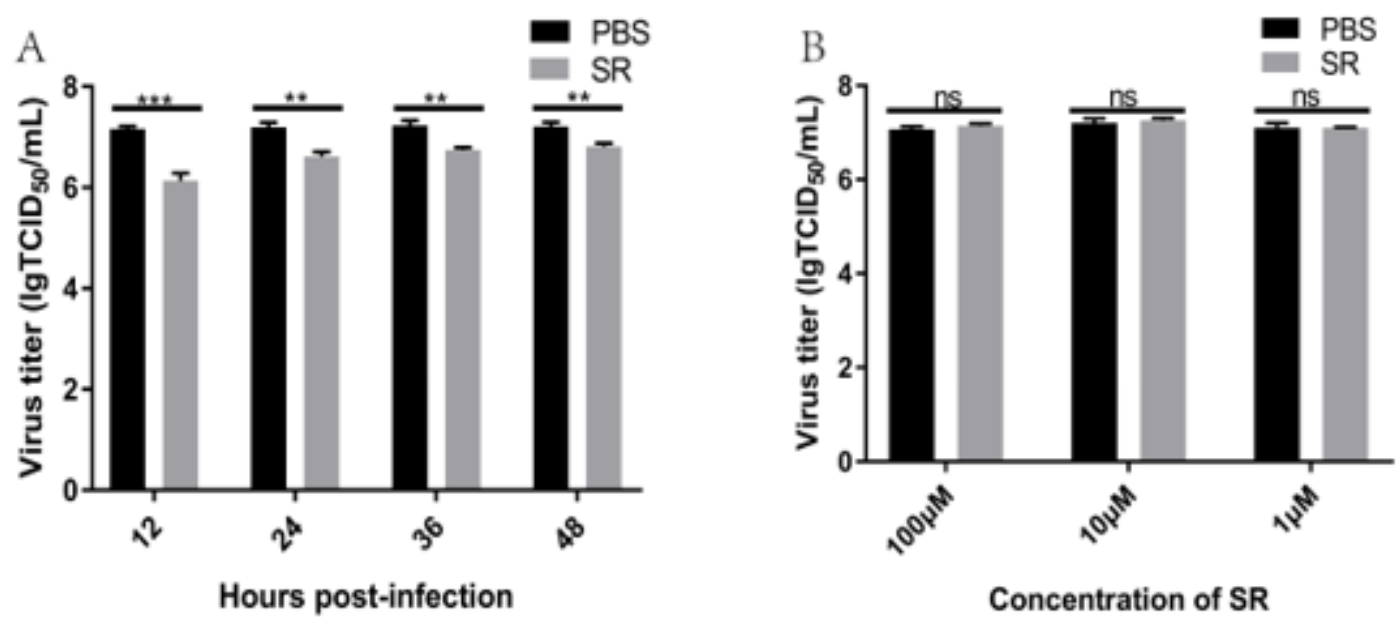

Figure 2

The inhibitory effects of SR on PRRSV. Marc-145 cells were treated with $100 \mu \mathrm{M}$ SR and infected with PRRSV and allowed to grow for $48 \mathrm{~h}(\mathrm{~A})$. PRRSV was incubated with different concentrations of SR for 2 $h$, and then, the TCID50 was determined by the Reed-Muench method (B). The data are shown as the means $\pm S D$ of three independent experiments $\left({ }^{*} p<0.05,{ }^{*} p<0.01,{ }^{*} *{ }^{*} p<0.001\right.$, and ${ }^{* \star * *} p<0.001$; ns, not significant).

A

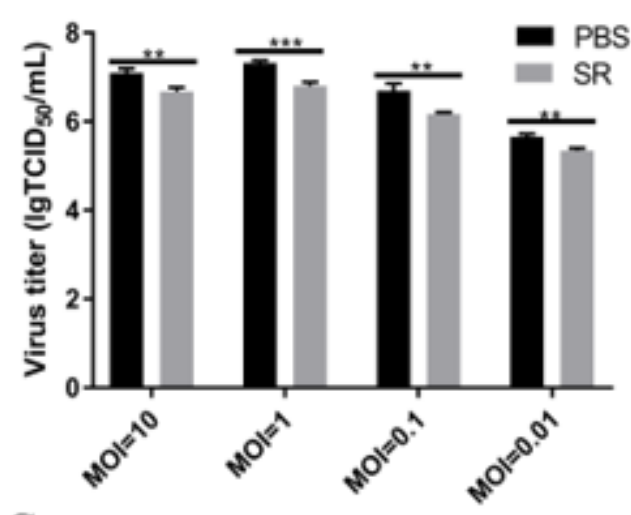

C

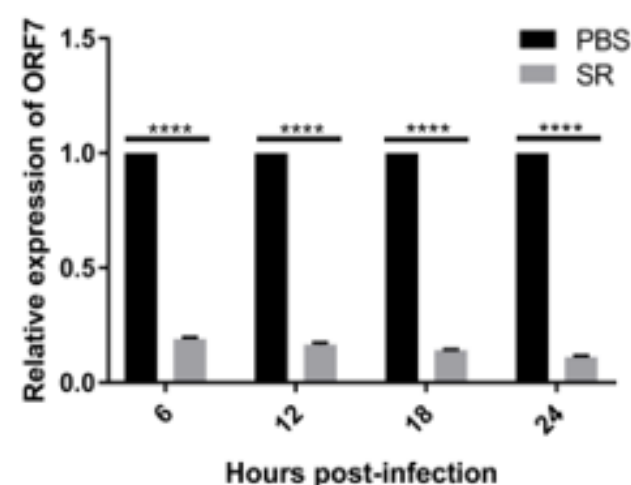

B

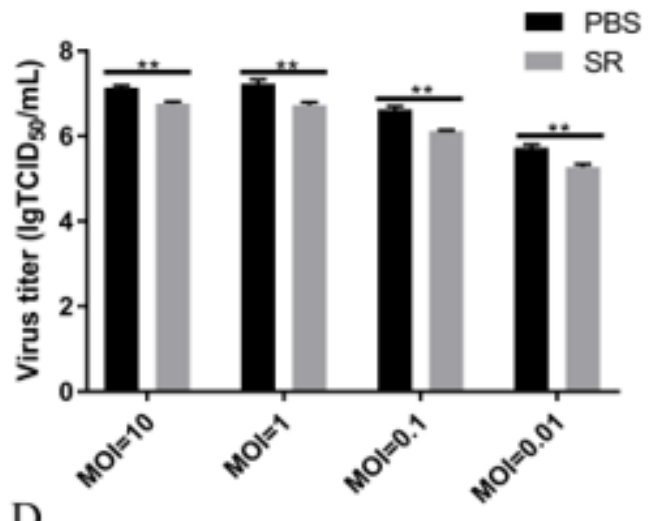

D

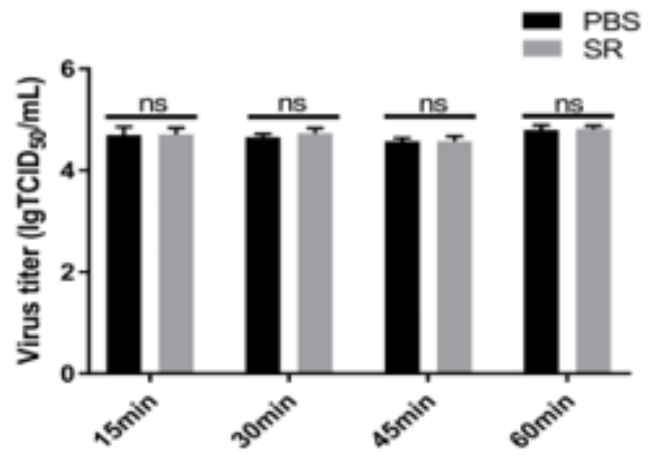

Minutes post-addition of SR

Figure 3 
SR inhibits virus invasion. Different MOls of PRRSV and $100 \mu \mathrm{M} \mathrm{SR}$ were incubated with the cells for $2 \mathrm{~h}$, and then, the non-adsorbed virus was removed. The virus titre was measured after incubation for $48 \mathrm{~h}$ (A). The PRRSV of different MOls was adsorbed on the cells. SR $(100 \mu \mathrm{M})$ was added to the cells onto which the virus had adsorbed, cultured for $3 \mathrm{~h}$, and then, SR was removed. The virus titre was measured after incubation for $48 \mathrm{~h}$ (B). Marc-145 cells were treated with SR $(100 \mu \mathrm{M})$ or PBS 2 hpi. The virus genome was determined at the specified time point (C). Marc-145 cells were treated with SR $(100 \mu \mathrm{M})$ or PBS $24 \mathrm{hpi}$. The virus titre was measured at the specified time point (D). Data are shown as the means $\pm S D$ of three independent experiments $\left({ }^{\star} p<0.05,{ }^{* \star} p<0.01,{ }^{\star \star *} p<0.001\right.$, and ${ }^{\star \star \star \star} p<0.001$; ns, not significant). 